\title{
EL APRENDIZAJE COLABORATIVO: UNA METODOLOGÍA EFICAZ PARA LA ENSEÑANZA DEL DERECHO PROCESAL LABORAL
}

\author{
Collaborative learning: \\ an effective technique for teaching Procedural Labour Law
}

\author{
Ana SÁnchez-Rubio \\ Profesora interina de Derecho Procesal \\ Universidad Pablo de Olavide de Sevilla \\ asanrub@upo.es
}

Orcid: $0000-0002-2027-8232$

Recibido: 17-07-2017

DOI: $10.1387 /$ lan-harremanak. 18415

Aceptado: 09-10-2017

\section{ABSTRACT}

El Grado en Relaciones Laborales y Recursos Humanos no posee un campo de conocimiento único sino que está configurado de manera híbrida, dado que engloba por igual conocimientos jurídicos y de gestión del personal. En concreto, en lo que respecta al Derecho procesal, el plan de estudios no contempla ninguna asignatura introductoria que permita a los alumnos establecer una primera toma de contacto con la terminología y la dinámica de esta disciplina. Por ello, a los estudiantes les resulta especialmente complejo enfrentarse a asignaturas como Derecho procesal laboral. Con el propósito de mitigar dicha sensación de dificultad, en las siguientes lineas se expondrán distintas técnicas docentes enfocadas hacia un modelo de clase colaborativa, en la que tanto los alumnos como el docente construyen la materia objeto de estudio al unisono, ya que hacer participe al alumno es el modo más eficaz de aprendizaje.

Palabras clave: educación universitaria; aprendizaje colaborativo; técnicas didácticas; Derecho procesal laboral. 
The Degree in Labour Relations and Human Resources has not a unique field of knowledge. On the contrary, this bachelor is configured in a hybrid way due to it includes both legal and management knowledge. Specifically, with respect to Procedural Law, the study program does not cover an introductory subject that allows students to stablish a first contact with the procedural terminology and the discipline. For these reasons, students find particularly complicated to face subjects like Procedural Labour Law. In order to mitigate this sensation of difficulty, the following pages explain different teaching techniques focus on a sort of collaborative lesson. In these sessions both students and professors build the matter together and their participation is a very effective way to learn.

Keywords: University education; collaborative learning; teaching techniques; Procedural Labour Law. 


\section{SUMARIO}

Sumario. 1. El alumno de Relaciones Laborales y el binomio enseñanzaaprendizaje. 2. Una aproximación conceptual al aprendizaje colaborativo. 3. El aprendizaje colaborativo en asignaturas jurídicas impartidas en el Grado de Relaciones Laborales y Recursos Humanos. 3.1. Formación del profesorado para aplicar de manera exitosa las técnicas de aprendizaje colaborativo. 3.2. El aprendizaje colaborativo a través de las TICs. 3.3. El aprendizaje colaborativo en los trabajos de fin de grado. 3.4. Aprendizaje colaborativo y educación inclusiva. 3.5. La rotación en las técnicas de aprendizaje colaborativo. 4. A modo de conclusión. 5. Bibliografía.

\section{El alumno de Relaciones Laborales y el binomio enseñanza- aprendizaje}

Cuando un profesor de Derecho ha de impartir clase en un grado distinto a la Licenciatura que él cursó suele explicar la materia a impartir dando por sabido conceptos, terminologías, funcionamientos del sistema, etc., que los alumnos desconocen. Esto es lo que sucede en el Grado de Relaciones Laborales y Recursos Humanos al momento de impartir la asignatura de Derecho Procesal Laboral. Y, esto es, precisamente, lo que provoca que muchos de los estudiantes de esta titulación sientan rechazo hacia las asignaturas pertenecientes a la rama del Derecho. Además, en el ámbito jurídico, parece que utilizar latinismos o una jerga sumamente artificiosa da al jurista un mayor renombre o un aire de sabiduría superior del que tiene quien utiliza un lenguaje claro y sencillo. Sin embargo, mi experiencia me dice que, al menos en lo que respecta a la docencia, esto provoca el efecto contrario: el receptor del mensaje, en este caso el estudiante, no admira más al profesor por este hecho, sino que pierde el interés en la asignatura porque se siente perdido desde el primer día.

Para paliar este efecto considero que debe sacarse partido de la faceta eminentemente práctica que tiene el Derecho Procesal, lo que debería facilitar el abandono de los tradicionales métodos de enseñanza basados en la presentación de diapositivas y el discurso del profesor, y optar por una enseńanza colaborativa, en la que el centro sea el papel participativo del alumno. En este sentido, sostiene Alonso (2014:126-127), que:

El Derecho Procesal permite, por su propio ámbito objetivo, una visualización de las implicaciones prácticas de muchos de los temas abordados. Y en esta misma lí- 
nea, propone los Moot Courts a modo de juicio simulados como la mejor opción, una simulación en la que todos los operadores jurídicos estén cubiertos por los alumnos, incluido, el Tribunal. Esta cuestión sitúa al profesor fuera de una situación supra-partes para convertirlo en una suerte de observador "casi» no participante, apostando así por otorgar margen de libertad al alumnado y estimulando de modo sano el ambiente competitivo para servir así de incentivo al alumnado.

Y ello, no solo permitiendo que intervenga para preguntar aquello que no sabe o que no entiende, sino para que lo construya él mismo.

Esas técnicas de innovación docente serán las que nos ocupen de aquí en adelante, pero antes de adentrarnos en su funcionamiento conviene recordar la importancia que revisten para el ámbito docente, pues la innovación educativa es en la actualidad un marco de referencia en la educación universitaria, que ha cobrado especial relevancia desde la creación del Espacio Europeo de Educación Superior. Este espacio propugna, entre otras cosas, una docencia centrada en el aprendizaje del estudiante, donde la misión del profesorado es la de guiar dicho proceso; una formación fundamentalmente práctica, orientada a la consecución de competencias y a la incorporación, de forma destacada, de las nuevas tecnologías en los procesos de aprendizaje del alumnado. En este contexto, los métodos y procedimientos docentes adquieren tanta importancia como los propios contenidos de las materias de estudio, en lo que respecta a la consecución de los objetivos de la educación general.

En concreto, en relación con el trabajo cooperativo, argumenta San José Cabezudo, et. al. (2008:386) que:

Ante el desafío que supone la adaptación al Espacio Europeo de Educación Superior (EEES), la capacidad de cooperar es, sin duda, una de las principales competencias que deben adquirir los futuros trabajadores de la sociedad del conocimiento y de la información. El aprendizaje colaborativo emerge, pues, como un paradigma prometedor en la educación universitaria que implica un nuevo rol del docente: facilitar a los estudiantes una ruta de aprendizaje a través de un proceso de descubrimiento colaborativo.

Ante este nuevo reto de docencia universitaria, el profesor se ha visto obligado a desarrollar nuevas estrategias didácticas, lo que implica rediseñar cómo enseña el docente y cómo aprende el alumno. En relación con ello afirma Kindelán (2013:28) que:

Este nuevo paradigma se centra en el discente, en el aprendizaje como eje en torno al cual gira todo el proceso educativo, haciendo a los estudiantes responsables de su formación, ofertándoles la posibilidad de elegir sus propias metas y las opciones que consideren más oportunas en función de sus necesidades y las demandas del mercado laboral. Mientras tanto, los profesores y formadores ya no son la única 
fuente de conocimiento, sino que funcionan como «tutores» que guían a los discentes en su camino único e individual hacia el conocimiento (cfr. European Commission, 2001, p. 6). Es en este ámbito donde la universidad ha de concebir una nueva forma de «enseñanza» que responda a este sistema basado en el aprendizaje.

Los métodos que están ganando posiciones en este contexto son aquellos a través de los cuales los estudiantes aprenden a pensar y a participar activa, reflexiva y creativamente. En tal sentido, las estrategias didácticas no se limitan a los métodos y las formas con los que se enseña, sino que además incluyen acciones que tienen en cuenta el repertorio de procedimientos, técnicas y habilidades que tienen los alumnos para aprender, acercando, así, los métodos de enseñanza a los métodos actuales de aprendizaje.

Así las cosas, la convergencia hacia este escenario educativo ha supuesto un desplazamiento de una educación centrada en la enseñanza hacia una educación centrada en el aprendizaje, a la vez que se exigen a los alumnos nuevas habilidades interpersonales que faciliten la interacción social y la cooperación. Todo ello implica introducir perspectivas diferentes en los métodos docentes y, en este sentido, las técnicas de colaboracionismo tienen una relevancia fundamental.

En lo que sigue se tratará de ofrecer un enfoque del aprendizaje colaborativo aplicado a las materias de contenido jurídico en el Grado de Relaciones Laborales. Con tal propósito se comenzará explicando al lector el concepto actual de aprendizaje colaborativo, para continuar proponiendo varias recomendaciones que, consideramos, incrementarían el valor didáctico de estas asignaturas y, en concreto, del Derecho Procesal Laboral. Por último, se finalizará con algunas conclusiones sobre la materia objeto de estudio.

\section{Una aproximación conceptual al aprendizaje colaborativo}

En una primera aproximación al concepto de aprendizaje colaborativo, y como paso previo a su aplicación al Grado de Relaciones Laborales, podría afirmarse que tal expresión remite a la existencia de una tarea grupal, es decir, de un objetivo que los distintos alumnos que trabajan conjuntamente deben alcanzar como grupo. Por tanto, la situación debe implicar no solo hacer cosas al unísono, sino afrontar y resolver una tarea o problema común $y$, como consecuencia, aprender juntos. De este modo, las metodologías de aprendizaje colaborativo se caracterizan porque el éxito y el fracaso se reparten entre los participantes, aportando cada alumno al grupo el beneficio de sus características más adecuadas. Es decir, el aprendizaje colaborativo es un proceso de construcción social en el que cada individuo aprende más de lo 
que aprendería por sí mismo debido a la interacción con otros miembros de su grupo.

En ello conviene Lucero (2003: 4), al destacar que:

El aprendizaje es un proceso individual que puede ser enriquecido con actividades colaborativas tendientes a desarrollar en el individuo habilidades personales y de grupo. El aprendizaje en ambientes colaborativos busca propiciar espacios en los cuales se dé la discusión entre los estudiantes al momento de explorar conceptos que interesa dilucidar o situaciones problemáticas que se desea resolver; se busca que la combinación de situaciones e interacciones sociales pueda contribuir hacia un aprendizaje personal y grupal efectivo.

Por consiguiente, puede convenirse en que los métodos de aprendizaje colaborativo comparten la idea de que los estudiantes trabajan juntos para aprender y son responsables del aprendizaje de sus compañeros tanto como del suyo propio. En estas situaciones la cooperación es superior a la competición y a la individualidad en cuanto al rendimiento y a la productividad de todos los participantes. Además, este hecho se muestra independiente de la naturaleza del contenido (ciencias sociales, ciencias de la salud, arquitectura, ingeniería, informática, etc.), de las diferencias de edades y de los niveles educativos. Y es que las técnicas de aprendizaje colaborativo mejoran la calidad de las estrategias docentes desde perspectivas generales. Por ejemplo, desarrollan estrategias de procesamiento de la información, favorecen el pensamiento crítico y constructivo y mejoran la capacidad de comunicación y expresión.

Respecto al concepto de aprendizaje colaborativo cabe puntualizar que, pese a que los términos aprendizaje "cooperativo" y "colaborativo» han sido utilizados indistintamente entre ellos, ambos están separados por una línea divisoria muy sutil: el aprendizaje cooperativo apunta a crear una estructura general de trabajo donde cada uno de los miembros es responsable de una tarea específica, en favor de organizaciones óptimas; por su parte, el aprendizaje colaborativo hace referencia al desarrollo cognitivo del individuo en la interacción con otros, cuidando especialmente la construcción colectiva del conocimiento y el desarrollo cognitivo de cada uno de los miembros del equipo. En realidad, se trata de dos conceptos complementarios para conseguir un aprendizaje en colaboración de calidad.

En concreto, mediante la utilización del aprendizaje colaborativo se ha demostrado que los estudiantes pueden tener más éxito que el propio profesor para hacer entender ciertos conceptos a sus compañeros. La razón de este hecho estriba en que los compañeros se relacionan en un entorno más cercano, en lo que respecta a su desarrollo cognitivo y a la experiencia en la materia de estudio. De esta forma, no solo el compañero que aprende se beneficia de la experiencia, 
sino también el estudiante que explica la materia a sus compañeros consigue una mayor comprensión. En este sentido, destaca Maldonado (2007: 265) el carácter social de aprender y sostiene que:

Ya el esquema que establecía al profesor como el que enseña y al estudiante como el que aprende de forma exclusiva, no tiene cabida. En su lugar se presenta el aprendizaje como un proceso social que se construye en la interacción no sólo con el profesor, sino también con los compañeros, con el contexto y con el significado que se le asigna a lo que se aprende.

En definitiva, de aquí en adelante aludiremos a técnicas de aprendizaje en las que el grupo de clase es considerado un agente social, cuya función esencial es enseñar, transmitir conocimientos, es decir, educar. Por lo tanto, el grupo deja de ser un mero entorno ambiental, como lleva siéndolo en la enseñanza tradicional, y pasa a cumplir la categoría de foco de interacción social, lo que exige que se tenga en cuenta la dinámica interna, los procesos de influencia recíproca y las habilidades sociales de los estudiantes.

\section{El aprendizaje colaborativo en asignaturas jurídicas impartidas en el Grado de Relaciones Laborales y Recursos Humanos}

La interdisciplinariedad que caracteriza al Grado en Relaciones Laborales y Recursos Humanos hace especialmente idóneo el uso de las técnicas de aprendizaje colaborativo. El principal motivo de ello es que habrá alumnos interesados más en el ámbito de gestión y otros más en el ámbito jurídico, por lo que una interacción entre todos ellos enriquecería su aprendizaje exponencialmente. No obstante, aplicar estas técnicas con las limitaciones de tiempo y de recursos materiales que tienen las universidades públicas puede ser algo inalcanzable. En lo que sigue se tratará de evidenciar tanto las carencias que dificultan su implantación como los remedios para suplir dichas carencias y los beneficios que he obtenido de su aplicación en los alumnos de la titulación que nos ocupa.

\subsection{Formación del profesorado para aplicar de manera exitosa las técnicas de aprendizaje colaborativo}

Entre algunos docentes universitarios aún existe la creencia reminiscente de que para saber enseñar basta con saber el contenido de las disciplinas y que, por consiguiente, la calidad de la enseñanza depende del nivel científico del profesorado y los recursos materiales disponibles. Sin embargo, la realidad ha demostrado que ello no es así. En este sentido, diversas investigaciones han concluido una baja correlación $(0.21)$ entre las valoraciones que los estudiantes hacen de 
sus profesores y la producción científica de éstos, llegándose a la conclusión de que la productividad científica y la eficacia docente son dimensiones independientes una de la otra (León y Latas, 2005: 46). Ante estos resultados, quizás, la todavía presencia de este pensamiento en el panorama universitario actual sea debida a que la universidad prefiere pertenecer al sistema científico en lugar de considerarse parte del sistema educativo, lo cual repercute negativamente en la actividad y en la calidad docente.

Si bien es cierto que la educación universitaria ha evolucionado mucho en este sentido, no es menos cierto que aún queda mucho por hacer en lo que respecta a la formación del profesorado en el ámbito estrictamente didáctico. Uno de los campos que necesitan un mayor desarrollo es el de las competencias que ha de adquirir el docente para aplicar de manera exitosa el aprendizaje colaborativo. En aras de alcanzar este propósito sería conveniente incluir en los planes de formación del profesorado diversas áreas psicosociales.

La primera de ellas englobaría, por ejemplo, trabajar en las habilidades de comunicación profesor-alumno, de percepción social, de formación de impresiones y de atribuciones causales o expectativas del profesor en su asignatura, es decir, en aspectos centrados en las destrezas del profesor como comunicador y responsable del grupo. La segunda área implicaría el aprendizaje por parte de los docentes en la formación y estructura de los grupos, en la cohesión grupal y en los conflictos más comunes dentro de este método de enseñanza, es decir, en los métodos y en las técnicas del aprendizaje colaborativo. No obstante, no es suficiente con crear un clima de trabajo adecuado y que el profesor esté instruido en la didáctica del aprendizaje colaborativo para que éste pueda ser aplicado correctamente, sino que es necesario trabajar en una tercera área: la relativa a la motivación de los alumnos. Esto es, se requiere que el docente esté formado en técnicas de coaching e inteligencia emocional para que incite a los alumnos a aprender en grupo. En relación con ello, señala Sánchez (2013: 175) que:

La investigación científica ha podido demostrar la alta efectividad del coaching como filosofía reflejada, tanto en su forma de gestionar el desarrollo de las personas, como en el proceso de cambio organizacional. De hecho, se empieza a contemplar que podría ser una herramienta clave para formar docentes líderes transformacionales, capaces de explorar y detectar las reales motivaciones de sus estudiantes. Docentes que puedan gestionar y enseñar a gestionar las creencias y emociones, las cuales, en definitiva, son el punto diferenciador entre la simple transmisión de conocimiento y la formación de excelentes seres humanos.

De nada sirve que el profesor sea un buen comunicador y que conozca a la perfección las metodologías del trabajo en equipo si no toma conciencia de cómo ha de dirigirse ese aprendizaje. Para que el aprendizaje colaborativo sea 
exitoso es imprescindible una actitud proactiva por parte de los alumnos, y procurar esa actitud es tarea del profesor. No obstante, ello no quiere decir que con la aplicación de estos métodos didácticos el profesor pase a un segundo plano, limitándose a guiar y acompañar a los alumnos en su proceso de aprendizaje. Eso es lo que perciben los alumnos en el aula y esa es la intención, que perciban que son los dueños de su aprendizaje. Pero el profesor tiene siempre un papel protagonista en este proceso, no solo en lo que se refiere a los contenidos sobre los que se trabaja sino a aspectos relativos al desarrollo personal de los alumnos.

Es por ello por lo que, trabajar la inteligencia emocional se convierte en una pieza esencial para el aprendizaje colaborativo. La misión de buscar la mejora del rendimiento individual a través del trabajo en grupo requiere de dotes psicológicas y pedagógicas que hemos de aprender los docentes antes de pretender que lo aprendan los alumnos. Además, para que del trabajo en grupo se obtengan resultados de calidad es necesario que los integrantes del mismo sepan adjudicar el rol que mejor casa con cada uno, comprender las limitaciones y los puntos fuertes de los otros e interactuar de la manera más equitativa posible. Si el docente encargado de supervisar estas competencias no las posee, o no las ha trabajado previamente, difícilmente va a conseguir llevar al grupo de alumnos en la correcta dirección.

Por todo lo expuesto, dedicar tiempo en clase a trabajar de forma prioritaria aspectos como la autocrítica o la comprensión del comportamiento de otros, aunque es una tarea prácticamente inabarcable en las escasas horas de docencia que tenemos asignadas actualmente, se convierte en algo casi tanto o más importante a la hora de aplicar el aprendizaje colaborativo que lograr explicar todo el temario.

\subsection{El aprendizaje colaborativo a través de las TICs}

En la Universidad los docentes tenemos a nuestra disposición los sistemas informáticos y tecnológicos más avanzados de nuestros tiempos, sin embargo, en la mayoría de los casos no se les saca el máximo rendimiento. Ello es debido, principalmente, a que a través de ellos se emplean métodos didácticos tradicionales. De este modo, el mayor uso que se hace del aula virtual se resume en adjuntar documentos, que posteriormente serán trabajados en una clase magistral, $\mathrm{y}$ en poner anuncios que informen al alumnado de cualquier noticia o incidencia (el cambio horario de una clase, el recordatorio de un seminario extraordinario, etc.).

En relación con el tema que nos ocupa, el aprendizaje colaborativo, tan solo los profesores más innovadores utilizan la herramienta de los foros de discusión. En este sentido, lo más frecuente es que el profesor responsable de la asignatura 
recomiende una lectura o lance una pregunta relacionada con el temario y establezca un espacio temporal para que los alumnos intercambien opiniones a través de la página del foro de la clase sobre dicho asunto. Sin duda, este instrumento facilita al alumno que no pueda/quiera acudir a clase la participación en la asignatura, e incluso llega a ser una herramienta sine qua non para cursos online. Las ventajas de estas herramientas son señaladas por García-Valcárcel et al. (2012: 162) al sostener que:

Las herramientas tecnológicas colaborativas pueden generar nuevos escenarios de aprendizaje derivados del impacto que la evolución de las comunicaciones ha tenidos sobre el modo en que trabajan las personas y sobre la estructura de las organizaciones. La contribución de las TIC al trabajo colaborativo es todavía reciente, pero sigue una marcada tendencia de crecimiento y desarrollo que vincula fuertemente la tecnología con la innovación y el constructivismo social.

Por ejemplo, en la asignatura de Derecho procesal se producen debates muy ricos, pues tras poner un caso de estudio cada alumno enfoca la solución desde una perspectiva diferente - posicionándose del lado del empresario o del trabajador-, y ello da lugar a un intercambio de opiniones que, en muchas ocasiones coinciden con los debates doctrinales, por lo que los alumnos aprenden la teoría porque ellos la están explicando, incluso sin ser conscientes de este hecho. En el ámbito jurídico esta técnica de innovación docente es conocida como el método del caso. Ordeńana (2014: 291) se refiere a ella apuntando que:

Este nuevo método, al igual que todos los denominados activos, cambia el rol del docente en la enseńanza universitaria: ya no es el artífice y protagonista del proceso de transmisión de conocimiento. Ahora, el aprendizaje se basa o centra en el estudiante, quien lejos de limitarse a copiar — sin estímulo y espíritu crítico alguno-, el contenido transmitido por el docente, construye su conocimiento. En términos psico-pedagógicos, se pasa de un aprendizaje conductivista a otro constructivista.

Otra vía para fomentar el aprendizaje colaborativo a través de las TICs, es acercarnos al entorno virtual en el que interactúan los alumnos, es decir, crear estos mismos foros fuera de las páginas de la universidad y trasladarlos a las redes sociales, como Facebook o Twitter. Ello nos permitirá estar más presentes en el día a día de los alumnos y compartir con ellos noticias de actualidad que les susciten interés y de las que se hable en las redes, en lugar de trabajar sobre autores o lecturas recogidos en una revista científica o en una monografía. Por ejemplo, con noticias jurídicas de actualidad o sentencias especialmente controvertidas.

Garcimartín (2014:19) también aboga por este método al sostener que:

Son muchas las razones que avalan este nexo entre actualidad informativa y Derecho: la habitual aparición de crónica jurídica en los medios de comunicación, 
su impacto social así como el hecho de que la prensa es el medio por el que el jurista conoce de forma más inmediata los problemas sociales a los que ha de responder y los cambios en el ordenamiento. Por eso uno de los objetivos de esta metodología es fomentar en los estudiantes un hábito de seguimiento crítico de las noticias jurídicas de actualidad, que les permita enfrentarse a los problemas que se plantean de forma razonada y analizar cómo son expuestos por los profesionales de la información.

\subsection{El aprendizaje colaborativo en los trabajos de fin de grado}

En buena parte de las titulaciones universitarias la implantación de una asignatura cuyo contenido consiste en la elaboración de un trabajo final ha significado una novedad. La mayoría de estos trabajos consisten en el desarrollo de un proyecto, más habitual en las carreras técnicas, o en la realización de un trabajo de investigación, opción más frecuente en las carreras de ciencias sociales. Ambas modalidades de trabajos de fin de grado suelen desarrollarse de manera individual, por lo que si durante los años de duración del grado se han estado trabajando técnicas de aprendizaje colaborativo, en uno de los momentos más decisivos para los estudiantes, todo este esfuerzo por parte de los docentes y de los propios alumnos no obtiene sus frutos: los alumnos vuelven a la enseñanza tradicional y a mirar únicamente por sí mismos y su calificación y no es así como se trabaja en el mercado laboral.

Precisamente por ello, queremos proponer aquí que los trabajos de fin de grado de los estudiantes de Relaciones Laborales se realicen en grupo. Para este cometido los grupos no deben tener muchos integrantes, entre 3 y 5 se situaría el tamaño óptimo, ya que a más estudiantes más probabilidades de que haya alguno de ellos que se beneficie del trabajo del resto sin aportar nada propio, bien porque se aproveche de la situación bien porque el grupo decida que no es una pieza clave y no tenga en cuenta sus contribuciones. Además, en grupos grandes es muy difícil que todos los estudiantes tengan la oportunidad de participar equitativamente y en parejas un estudiante puede dominar.

Lo esencial es que cada alumno desempeñe un rol vital dentro del grupo, de manera que el trabajo no pueda seguir sin él. Para el cumplimiento de este objetivo será el profesor el que decida la composición del mismo, según la evolución de los alumnos a lo largo de los años del grado. También será cometido del profesor insistir en que trabajar en equipo no significa repartirse el trabajo, sino que todos han de participar de la totalidad de la investigación o del proyecto, creando una sinergia. Una de las maneras de controlar que el trabajo ha sido realizado por todos y entre todos los integrantes de cada grupo es que el presidente del tribunal que ha de evaluar el trabajo elija al azar y en el mismo acto de la defensa quien o quienes lo expondrán. 
Además, estos trabajos no tienen por qué consistir siempre en una investigación de un punto del programa docente. En este sentido, en la titulación de Relaciones Laborales sería especialmente importante que, en función de la especialidad por la que opte el alumno el TFG sea la redacción de una sentencia, la elaboración de nóminas, la creación de un convenio colectivo, etc. Es decir, crear un aprendizaje colaborativo con subgrupos de intereses comunes que favorezcan la especialización de los estudiantes.

\subsection{Aprendizaje colaborativo y educación inclusiva}

Una de las grandes ventajas del aprendizaje colaborativo es que todos los alumnos de un equipo deben tener la oportunidad y la "obligación» de expresar su punto de vista y discutir y dialogar con los demás hasta llegar a un acuerdo. Esta gran ventaja se convierte en un enorme beneficio para los alumnos que, por diversos motivos, no se sienten integrados con plenitud en la clase a la que asisten. Dicha falta de integración puede deberse a razones muy diferentes: falta de habilidades sociales, ausencia de motivación académica, marginación por parte de la mayoría de los alumnos de la clase o, incluso, padecer algún tipo de discapacidad que impida al estudiante adaptarse al ritmo del grupo.

La utilización de grupos colaborativos en clase es un mecanismo ideal para aprovechar el potencial del aprendizaje entre compañeros, especialmente si esta heterogeneidad posibilita una integración social. Con relación a ello, se ha comprobado que el uso de grupos en clase aumenta la probabilidad de que los estudiantes se reúnan fuera de la universidad para continuar estudiando juntos o realizar actividades de ocio. Ello repercute directamente en un incremento de la motivación de los estudiantes que no se sentían cómodos en clase y, consecuentemente, en un descenso del absentismo.

En estas situaciones se acentúa aún más la necesidad de que el profesor desarrolle sus habilidades de inteligencia emocional para que estimule al estudiante desmotivado e inserte al tímido, e incluso al discapacitado, teniendo en cuenta sus limitaciones. Para ello recomendamos, en un principio, la creación de grupos de trabajo heterogéneos y, posteriormente, rotatorios. Esto es, los equipos deben estar compuestos al principio del curso por personas que no tengan características ni intereses comunes. Pero, este equipo debe diluirse cuando, pasadas unas clases, ya hayan alcanzado la confianza y cohesión esperada y deben formarse nuevos equipos, de manera que se promueva una rotación que permita que los estudiantes hayan trabajado con todos sus compañeros al final del curso.

Por último, para que el aprendizaje colaborativo funcione como estrategia para la inclusión de alumnos es fundamental retomar las diferencias entre cola- 
boración y cooperación, y apostar por que las dos metodologías de aprendizaje interactúen al unísono. Es decir, para lograr una universidad inclusiva los docentes debemos trabajar en que se practique la cooperación y no solo la colaboración. Ello implica que no basta con que los alumnos trabajen juntos, sino que han de ayudarse mutuamente en sus respectivas carencias.

\subsection{La rotación en las técnicas de aprendizaje colaborativo}

El aprendizaje colaborativo puede practicarse a través de múltiples técnicas que tienen como denominador común la creación de grupos de trabajo. Entre las más destacadas se encuentran, el brainstorming ${ }^{1}$, la técnica Philips $6 / 6^{2}$, el jigsaw ${ }^{3}$, la pecera o la rejilla. En particular, merecen ser destacados los resultados obtenidos tras la aplicación de estas dos últimas técnicas que parecen ser las menos utilizadas, ya que cuando las implanté en clase los estudiantes me aseguraron no haberlas trabajado antes.

Por un lado, la pecera se trata de una técnica en la que un círculo exterior de estudiantes se sienta alrededor de otro círculo interior más pequeño de alumnos. Los estudiantes del círculo interior participan en un diálogo en profundidad, mientras los que están en el círculo exterior examinan lo que se dice y cómo se dice. Lo que se pretende es que los estudiantes del círculo exterior critiquen el contenido, la lógica y la interacción del círculo interior, por lo que es un buen método de evaluación P2P4 . Esta técnica la he llevado a cabo con resoluciones de judiciales contradictorias sobre la misma materia, lo que ha dado lugar a que una parte de los alumnos apoyen un pronunciamiento y otra parte el pronunciamiento contrario. De este modo, el círculo interior ha interactuado activamente,

1 Conviene recordar que el brainstorming, cuya traducción literal es «lluvia de ideas» es una técnica de creatividad en grupo, donde los miembros del grupo aportan, durante un tiempo previamente establecido el mayor número de ideas posibles sobre un tema o problema determinado. Interesa, en primer lugar, la cantidad de ideas; conviene que las aportaciones sean breves, que nadie juzgue ninguna, que se elimine cualquier crítica o autocrítica y que no se produzcan discusiones ni explicaciones. Sin embargo, tras esta recepción de ideas sí que se elegirán solo las de mayor calidad.

2 Puede decirse, resumidamente, que esta técnica consiste en la división de la clase en diversos grupos de seis personas a los que se les da la oportunidad de hablar durante un minuto cada uno, de manera que en seis minutos hayan participado todos los integrantes y pueda extraerse una conclusión común.

3 Esta metodología también conocida como rompecabezas, es una técnica de aprendizaje colaborativo que crea una interdependencia positiva grupal, ya que cada equipo de trabajo solo tiene una parte de la información sobre la que se va a trabajar, que es diferente a la que tienen otros grupos, de modo que se hace necesaria una colaboración entre los miembros de un mismo grupo y entre los de grupos diferentes para alcanzar a comprender la información proporcionada como un todo.

4 Peer to peer (P2P) es una corrección entre iguales, dónde un estudiante corrige los trabajos de otros compańeros que se le asignen como parte del aprendizaje. Al ponerse en el sitio de un redactor y de un corrector, se pretende que el estudiante sea más consciente de todos los aspectos de su aprendizaje y asimile los conocimientos de una forma más crítica. 
mientras que los alumnos del círculo exterior con su posición de escucha se han nutrido de las opiniones del resto y han creado la suya propia. Al finalizar el debate del círculo interior, los del círculo exterior han criticado los errores de contenido o de expresión de quienes habían dialogado.

Por otro lado, cabe resaltar la técnica de la rejilla. En este método de aprendizaje colaborativo cada grupo de alumnos tendrá una información diferente del mismo tema a tratar, y las personas integrantes de los mismos van a ir rotando, de manera que la misma persona va a formar parte primero de un grupo y después de otro. De este modo se pretende que la información pase de un grupo a otro por medio del cambio de miembros de grupos, sin proporcionarle el informe correspondiente. Se trata de una técnica claramente dirigida a trabajar las habilidades de memoria y comunicación e integración de los alumnos.

Cada una de las técnicas mencionadas potencian habilidades diferentes de los estudiantes, por ello, cuando se opta por el aprendizaje colaborativo los docentes debemos aplicar todas las metodologías que conozcamos a lo largo de un curso. Con esta propuesta se pretende que los alumnos desarrollen al máximo sus capacidades de aprendizaje y, especialmente, de aprendizaje en grupo. Esta rotación ha de ser efectuada junto con la rotación de miembros de grupos citada supra. Además, con la variación de técnicas y de integrantes de equipos comprobaremos si cada uno de ellos mantiene el mismo rol, es decir, si el alumno que era líder en el brainstorming sigue siéndolo con la técnica del jigsaw o ha perdido este papel al no tener habilidades sociales para interaccionar con personas de fuera de su propio grupo.

El motivo por el que abogamos que se debe potenciar esta rotación reside en que la misma da lugar a situaciones que el estudiante va a experimentar en el mundo laboral, cuando cambie de empresa o cuando entren nuevos trabajadores en la empresa de la que forma parte. Y, precisamente, la principal tarea del docente universitario es preparar al alumno, profesional y personalmente, para su inserción laboral.

\section{A modo de conclusión}

Como ha quedado de manifiesto, en virtud de estas nuevas técnicas didácticas, no solo el profesor, sino también el alumno es responsable de su aprendizaje debiendo tomar un papel activo y participativo. Ello acerca al alumno a materias áridas, como puede ser la del Derecho procesal, y fomenta el interés de los estudiantes en las asignaturas de esta disciplina, no tanto por su contenido como por la forma de enseñarlos y de aprenderlos. Es ahí donde reside el beneficio que ofrece esta técnica docente. 
En concreto, tras aplicar esta metodología en la impartición de la asignatura de Derecho Procesal Laboral en el Grado de Relaciones Laborales y Recursos Humanos los resultados de un curso para otro fueron muy superiores. De sobra es conocido que pueden haber influido otras variables, como un curso compuesto de alumnos más aplicados o más interesados en la rama jurídica, pero lo cierto es que los beneficios de utilizar esta técnica no fueron únicamente académicos. En el aspecto social también se materializó el éxito de este tipo de aprendizaje pues, gracias al trabajo en equipo, un alumno aislado por sus compañeros por ser diferente a ellos — padecía el síndrome de Asperger-logró integrarse en el grupo de clase, lo que redundó en un mayor rendimiento académico también para el resto de asignaturas.

Ahora bien, para poder obtener estos buenos resultados de manera continuada en el tiempo se hace necesario demandar a las universidades mayor disponibilidad de espacios, recursos, medios y horarios para adaptar la enseñanza universitaria a la metodología del aprendizaje colaborativo. Y, sobre todo, se hace necesario un cambio de actitud por parte del profesorado universitario hacia la docencia, consistente en una mayor preocupación por los procesos de enseńanza-aprendizaje y por el alumno. La Universidad y las instituciones vinculadas a la misma deben apoyar al profesorado para que pueda adquirir la práctica suficiente. De igual manera, debe plantearse la necesidad de proporcionar a sus profesores la información y los recursos necesarios para la correcta aplicación de las metodologías de aprendizaje colaborativo.

\section{Bibliografía}

Alonso Salgado, Carlos (2014): «Simulación de roles y juego de Edwards para la evaluación de competencias en el Derecho procesal», en Reduca (Derecho). Serie Derecho Procesal, 5.

García-Valcárcel Muñoz-Repiso, Ana; Hernández Martín, Azucena; Recamán Payo, Adriana (2012): «La metodología del aprendizaje colaborativo a través de las TIC: una aproximación a las opiniones de profesores y alumnos», en Revista Complutense de Educación, 23-1.

Garcimartín, Regina (2014): «Análisis crítico de actualidad jurídica como método docente", en Reduca (Derecho). Serie derecho procesal, 5.

Kindelán, María Paz (2013): «Una perspectiva sobre el binomio enseñanza-investigación en la universidad del siglo XXI», en Revista Complutense de Educación, 24-1.

León del Barco, Benito y Latas Pérez, Carlos (2005): «Nuevas exigencias en el proceso de enseñanza aprendizaje del profesor universitario en el contexto de la convergencia europea: la formación en técnicas de aprendizaje cooperativo", en Revista Electrónica Interuniversitaria de Formación del Profesorado, 8.

LuCERo, María Margarita (2003): «Entre el trabajo colaborativo y el aprendizaje colaborativo», en Revista Iberoamericana de Educación, 4. 
Maldonado PÉrez, Marisabel (2007): «El trabajo colaborativo en el aula universitaria», en Revista de Educación, 13-23.

Ordeñana Gezuraga, Ixusko (2014): «La enseñanza-aprendizaje de las bases del Derecho procesal civil mediante el case method: una experiencia y su lectura crítica», en Reduca (Derecho). Serie derecho procesal, 5.

SÁNCHEZ-Teruel, David (2013): «El coaching pedagógico dentro del sistema educativo: innovando procesos», en Revista Intercontinental de Psicología y Educación, 15-2.

San José Cabezudo, Rebeca; Antón Martín, Carmen; Camarero Izquierdo, Carmen; De la Cuesta de Diego, Borja; Gutiérrez Cillán, Jesús; Hernández Carrión, Carlos; Rodríguez Escudero, Ana Isabel; Rodríguez Pinto, Javier; San Martín Gutiérrez, Sonia; Silva García, Rebeca (2008): «Nuevos retos en innovación docente: uso de foros y aprendizaje colaborativo", en Guillarte Martín-Calero, Cristina (coord.), Innovación docente: docencia y TICs, Universidad de Valladolid, Valladolid. 